\title{
An Instantaneous Normal Mode Description of Relaxation in Supercooled Liquids
}

\author{
T. Keyes \\ G. V. Vijayadamodar \\ Ulrich Zurcher \\ Cleveland State University, u.zurcher@csuohio.edu
}

Follow this and additional works at: https://engagedscholarship.csuohio.edu/sciphysics_facpub

Part of the Physics Commons

How does access to this work benefit you? Let us know!

Publisher's Statement

(C) 1997 American Institute of Physics.

\section{Repository Citation}

Keyes, T.; Vijayadamodar, G. V.; and Zurcher, Ulrich, "An Instantaneous Normal Mode Description of Relaxation in Supercooled Liquids" (1997). Physics Faculty Publications. 274.

https://engagedscholarship.csuohio.edu/sciphysics_facpub/274

This Article is brought to you for free and open access by the Physics Department at EngagedScholarship@CSU. It has been accepted for inclusion in Physics Faculty Publications by an authorized administrator of

EngagedScholarship@CSU. For more information, please contact library.es@csuohio.edu. 


\title{
An instantaneous normal mode description of relaxation in supercooled liquids
}

\author{
T. Keyes, G. V. Vijayadamodar, and U. Zurcher \\ Department of Chemistry, Boston University, Boston, Massachusetts 02215
}

(Received 25 October 1996; accepted 16 December 1996)

\begin{abstract}
Relaxation in supercooled liquids is formulated from the instantaneous normal modes (INM) point of view. The frequency and temperature dependence of the unstable, imaginary frequency lobe of the INM density of states, $\left\langle\rho_{u}(\omega, T)\right\rangle$ (for simplicity we write $\omega$ instead of $i \omega$ ), is investigated and characterized over a broad temperature range, $10 \geqslant T \geqslant 0.42$, in the unit density Lennard-Jones liquid. INM theories of diffusion invoke Im- $\omega$ modes descriptive of barrier crossing, but not all imaginary frequency modes fall into this category. There exists a cutoff frequency $\omega_{c}$ such that modes with $\omega<\omega_{c}$ correspond to "shoulder potentials," whereas the potential profiles include barrier-crossing double wells for $\omega>\omega_{c}$. Given that only modes with $\omega>\omega_{c}$ contribute to diffusion, the barrier crossing rate, $\omega_{h}$, and the self diffusion constant $D$, are shown to be proportional to the density of states evaluated at the cutoff frequency, $\left\langle\rho_{u}\left(\omega_{c}, T\right)\right\rangle$. The density of states exhibits crossover behavior in its temperature dependence such that the exponential $T$-dependence of $D(T)$ crosses over from Zwanzig-Bassler $\exp \left(-E^{2} / T^{2}\right)$ behavior at low $T$ to Arrhenius $\exp (-E / T)$ behavior at high $T$; the exponential may be too weak to be observed, in which case $D(T)$ is a power law. Based on the properties of LJ, a general INM description of strong and fragile liquids is presented, with a physical interpretation in terms of the "landscape" of the potential energy surface. (C) 1997 American Institute of Physics.
\end{abstract}

[S0021-9606(97)02711-6]

\section{INTRODUCTION}

A new paradigm of liquid state dynamics is emerging based upon instantaneous normal modes (INM). ${ }^{1}$ The INM are the eigenfunctions of the force constant matrix, or Hessian, for a representative configuration; ultimately a configuration average is taken. In order to exploit the INM viewpoint it is useful to represent the liquid as a point in a $3 N$ dimensional configuration space, and to think of the dynamics as motion of this point over the total potential energy surface $U$. The essential features of the $U$-surface are the global crystal minimum, the local minima and associated basins or wells, and the saddle barriers connecting the wells. Thus the system point may be considered ${ }^{2}$ to move from well to well via a series of barrier crossings. During its sojourns in the wells the liquid executes harmonic oscillations, which are interrupted by hopping; correspondingly, time correlation functions are expressed ${ }^{3}$ as superpositions of damped harmonic oscillator contributions.

Near the bottom of a well, the INM are the conventional lattice vibrations of a disordered lattice, with real frequencies characterizing the upward curvature of the well. On the other hand, during barrier crossing, $U$ has downward curvature in some directions, and the modes associated with those directions have imaginary frequencies. Thus the configuration averaged INM density of states, $\langle\rho(\omega)\rangle$, has two contributions, $\langle\rho(\omega)\rangle=\left\langle\rho_{s}(\omega)\right\rangle+\left\langle\rho_{u}(\omega)\right\rangle$, where "s" refers to the real frequency "stable" modes and " $u$ " to the imaginary frequency "unstable" modes. Undamped unstable modes exhibit exponential growth, indicating the limits of a purely harmonic approach to liquids. We have suggested ${ }^{4}$ that the lowest Im- $\omega$ modes do not correspond to barriers. Investigating the potential energy profiles along the INM normal co- ordinates, Bembenek and Laird ${ }^{5}$ verified this suggestion, and argue that only the barrier crossing modes should be called unstable. Nevertheless, we will keep our " $u$ " notation for all Im- $\omega$ modes. Usually ${ }^{1}$ imaginary INM frequencies are treated as negative frequencies and $\left\langle\rho_{u}(\omega)\right\rangle$ is plotted along the negative real frequency axis. In this article, which concerns the unstable modes only, we simply use positive $\omega$ to denote $i \omega$. Natural units are used in all Lennard-Jones fits and simulation data; $T$ denotes temperature in units of the well depth $\epsilon$, density is $(N / V) \sigma^{3}, \sigma$ is the hard core diameter, frequency is $\omega \tau, \tau$ is the $\mathrm{LJ}$ time. For Argon, $\epsilon / K_{B}=119.8 \mathrm{~K}$, $\sigma=3.405 \AA$ and $\tau=2.18$ ps. Temperature and energy are interchangeable in these units.

The INM formalism is particularly appealing for supercooled liquids. As $T$ is decreased, the lifetime $\tau$ of the system point in the wells increases, the system stays closer to the bottom of the wells, and the barrier hopping rate $\omega_{h}=\tau^{-1}$ decreases. The more deeply supercooled the liquid, the closer it is to an amorphous solid, and the better a candidate it is for an INM description. This is a fortunate circumstance, since explanation of the behavior of supercooled liquids constitutes one of the most challenging areas of theoretical physical chemistry. However, despite the growing body of work on INM in liquids, little has been done so far on developing an INM theory for the signature properties ${ }^{6}$ of supercooled liquids, the most important being the strong exponential temperature dependence of relaxation times. In this paper, we present the first step in such a theory, an INM treatment and interpretation of the temperature dependence of the self diffusion constant, $D$.

The theory rests upon the detailed $\omega, T$ dependence of $\left\langle\rho_{u}(\omega)\right\rangle$ over a broad temperature range $10 \geqslant T \geqslant 0.42$, which 
we have determined for the unit density LJ liquid. However, we are only interested in LJ insofar as it reveals a form for $\left\langle\rho_{u}(\omega)\right\rangle$ which we will assume holds for more complex liquids. Thus we obtain a general INM formalism for the description of relaxation in supercooled liquids. Within this scheme, as $T$ increases from the vicinity of the glass transition $T_{G}, D(T)$ can change from Zwanzig-Bassler ${ }^{7}$ [' $\mathrm{ZB}$,', $\left.\exp \left(-E^{2} / T^{2}\right)\right]$ to Arrhenius $[\exp (-E / T)]$, to power law. The ranges of the different characteristic $T$-dependence, and the possibility that a given $T$-dependence will be visible, are determined by the parameters in the model. Depending on the values of the parameters, a rich variety of $T$-dependences are possible, including those 6 of "strong", and "fragile", liquids.

\section{INM THEORY OF SUPERCOOLED LIQUID DYNAMICS}

Zwanzig proposed ${ }^{3}$ that harmonic oscillations in the local minima of the $N$-body potential are randomized by barrier crossing, with the result for the velocity correlation function,

$$
C(t)=T \int d \omega \rho_{q}(\omega) \cos (\omega t) \exp \left(-\omega_{h} t\right), \neg
$$

where $\rho_{q}(\omega)$ is the quenched density of states representative of the local minima. Integration yields the diffusion constant; in the deeply supercooled liquid, where $\omega_{h} \rightarrow 0$,

$$
D(T) / T=\left\langle\omega^{-2}\right\rangle \omega_{h} \cdot \neg
$$

We proposed $^{8}$ that $\omega_{h}$ could be calculated from the imaginary frequency INM density of states, $\left\langle\rho_{u}(\omega)\right\rangle$. The physical reason is ${ }^{4,8}$ that $\left\langle\rho_{u}(\omega)\right\rangle$ may be written,

$$
\left\langle\rho_{u}(\omega, T)\right\rangle=a(T) G(\omega, T)\left\langle\rho_{u}(\omega, T=\infty)\right\rangle,
$$

where $G(\omega, T)$ is the averaged Boltzmann factor for barriers with curvature $\omega,\langle\exp (-\beta E)\rangle_{\omega}$; clearly, $\left\langle\rho_{u}(\omega, T)\right\rangle$ must be proportional to the Boltzmann probability of visiting a barrier with curvature $\omega$. Similarly, for barriers with a given $\omega$, all exponential $T$-dependence of the hopping rate is determined on average by this same quantity, and the total rate is obtained by summing over all frequencies. In short, $\omega_{h}$ may be expressed as a frequency integral of $\left\langle\rho_{u}(\omega)\right\rangle$, [Eq. (26) of Ref. 4],

$$
\omega_{h}=m c_{2} \int d \omega\left(\omega / \omega_{m}\right) f\left(\omega, \omega_{m}\right)(a(T))^{-1}\left\langle\rho_{u}(\omega)\right\rangle,
$$

where $m$ is the averaged number of minima connected to a barrier, $a(T)$ is the $\omega$-independent multiplicative factor obtained in a fit of the $\omega, T$ dependence of $\left\langle\rho_{u}(\omega)\right\rangle$, and $c_{2}$ determines the $T$-dependence of $a(T), a(T)=c_{1}-c_{2} f_{u}(T)$; $f_{u}(T)$, the "fraction of unstable modes," is the frequency integral of $\left\langle\rho_{u}(\omega)\right\rangle$. The hopping rate depends, irrespective of INM theory, upon the formula used for the rate of crossing a single well-characterized barrier. The quantity $f\left(\omega, \omega_{m}\right)$ is the pre-exponential factor in the chosen rate law. In Ref. 4 we used transition state theory, with $f\left(\omega, \omega_{m}\right)=\omega_{m} /(2 \pi)$.

Combination of Eqs. (2.2) and (2.4) yields an INM theory of self diffusion in supercooled liquids. Fits per- formed in Ref. 4 determined everything but the parameter $m$ in Eq. (2.4) for the unit density LJ liquid; for simplicity we chose $m=2$, corresponding to a one-dimensional "reaction coordinate." With $m=2$ and with the approximate quenched $\rho_{q}(\omega)$, we obtained quantitatively accurate diffusion constants for $1.25>T>0.66$; the melting temperature is $\sim 1.8$, and our data go down to $T=0.42$.

The functional form determined for the supercooled liquid is

$$
\left\langle\rho_{u}(\omega, T)\right\rangle=a(T) \omega \exp \left(-c \omega^{4} / T^{2}\right) .
$$

$$
\text { ( supercooled liquid) }
$$

Note that, for a single $\omega$, Eq. (2.5) has ZB T-dependence. However, the expression obtained ${ }^{4}$ for $D$ was $D(T) / T \sim T^{3 / 2}$, power law behavior. The integrand in Eq. (2.4) has exponential $T$-dependence at a single $\omega$, but only a power law remains after the integration. We consider this to be a crucial point. In the INM formalism the hopping rate is expressed as a frequency integral, which sums the contributions of the barriers with different curvatures $(\omega)$. The barriers with a particular curvature may contribute exponential $T$-dependence to $D$, but the sum over all barriers is the ultimate arbiter of how $D$ depends on $T$. A parallel argument may be phrased in terms of the activation energy; a barrier with a given $E$ makes an Arrhenius contribution to $\omega_{h}$, but the sum over contributions from all $E$, performed with use of the distribution of barrier heights $g(E)$, can have almost any $T$-dependence. The exponential $T$-dependence of $\left\langle\rho_{u}(\omega, T)\right\rangle$ is just that of the averaged Boltzmann factor, $G(\omega, T)$; for supercooled LJ,

$$
G(\omega, T) \sim \exp \left(-c \omega^{4} / T^{2}\right) . \neg
$$

For $T<0.66$, the simulated $D / T$ begin to fall below the power law, suggesting that exponential $T$-dependence-the dominance of activated barrier crossing-was beginning to set in. How can this be found in the theory? The answer lies in implementing the cutoff described in the introduction, recognizing that modes with $\omega<\omega_{c}$ do not correspond to barriers and should be excluded from $\omega_{h}$ by putting a lower cutoff $\omega_{c}$ on the integral in Eq. (2.3). Now, the original power law is recovered at high temperature, but ZB behavior 'survives' the cutoff integration and manifests itself at low $T$,

$$
\left.D(T) / T \sim T^{3 / 2} \exp \left(-c \omega_{c}^{4} / T^{2}\right) \sim T^{3 / 2} G\left(\omega_{c}, T\right) ;\right\urcorner
$$

a fit to the data gave $\omega_{c} \sim 5$. Clearly, the lower cutoff is essential to an INM description of the exponential $T$-dependence of dynamical quantities in supercooled liquids. Equation (2.7) is a specific example of one of our most important results; in general, we suggest that

$$
D(T) \sim G\left(\omega_{c}, T\right) \text { (exponential } T \text {-dependence only). }
$$

The use of a cutoff was ${ }^{5}$ put on a sound basis by Bembeneck and Laird. We have found similar behavior, and some analytical results, in a calculation ${ }^{9}$ based on application 


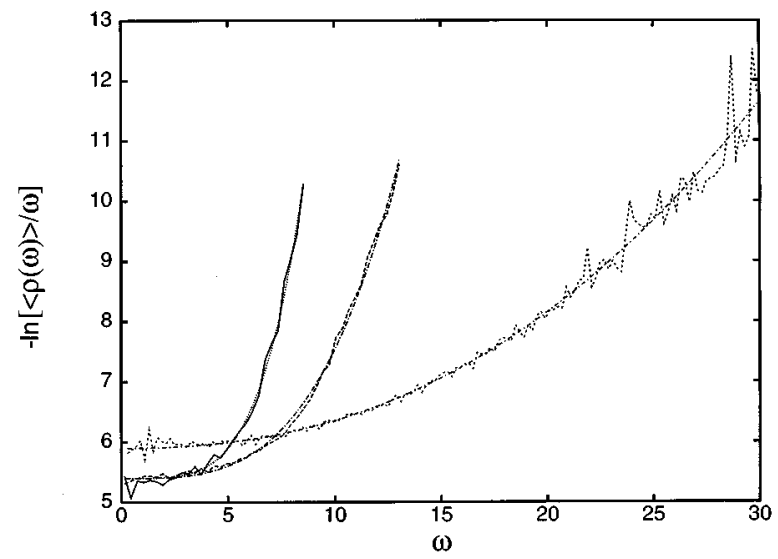

FIG. 1. Frequency dependence of unstable density of states expressed by $-\ln [\langle\rho(\omega)\rangle / \omega]$ at three temperatures, raw data and final fits (smooth lines) both shown; $T=0.50$ (sharpest rise), $T=1.0, T=5.0$ (weakest rise).

of the "soft potential model" to liquids. Bembeneck and Laird refer to the double well imaginary $\omega$ modes as the true "unstable modes"; their density of unstable modes has a lower cutoff $\omega_{c}$, from which it rises smoothly-it is not just the cutoff $\left\langle\rho_{u}(\omega)\right\rangle$. Nevertheless, a cutoff $\left\langle\rho_{u}(\omega)\right\rangle$ reproduces all the important physical and qualititative features of the INM description of supercooled liquids, and we will use it in the following.

Another piece of the puzzle has recently fallen into place. Vijayadamodar and Nitzan, ${ }^{10}$ studying the normal rather than the supercooled unit density LJ liquid, found

$$
\left\langle\rho_{u}(\omega)\right\rangle=a(T) \omega \exp \left(-c \omega^{2} / T\right), \neg \quad \text { (normal liquid) }
$$

i.e., $G(\omega, T) \sim \exp \left(-c \omega^{2} / T\right)$ and Arrhenius $T$-dependence for $D(T)$ via Eq. (2.8). With $\left\langle\rho_{u}(\omega)\right\rangle$ described by Eqs. (2.9) and (2.5) at high and low temperatures, respectively, the INM theory will yield a crossover from Arrhenius to ZB $T$-dependence at some intermediate temperature. Since the presence or absence of such a crossover is what distinguishes ${ }^{6}$ "fragile" from "strong" liquids, we now have an INM window upon this most fundamental characterization of supercooled liquids. A fit of $\left\langle\rho_{u}(\omega)\right\rangle$ for unit density LJ over the range $10>T>0.42$, which reproduces the two limiting forms, will be reported in the next section and used in the calculation of $\omega_{h}$. It is not the behavior of LJ that is particularly interesting, but the suggestion of a general model of exponential $T$-dependence applicable to all supercooled liquids.

\section{THE FUNCTIONAL FORM OF THE IMAGINARY FREQUENCY DENSITY OF STATES}

We have tried several functional forms with the indicated limiting behavior for $\left\langle\rho_{u}(\omega ; T)\right\rangle$ in unit density LJ for $10.0>T>0.42$; our simulation method for the supercooled points is described in Ref. 4. The obvious try of an exponent which is a sum of an $\omega^{2}$ term and an $\omega^{4}$ term is unsatisfactory because ${ }^{10} \omega^{2}$ is the high frequency behavior of the exponent. Various algebraic functions which are correct in this

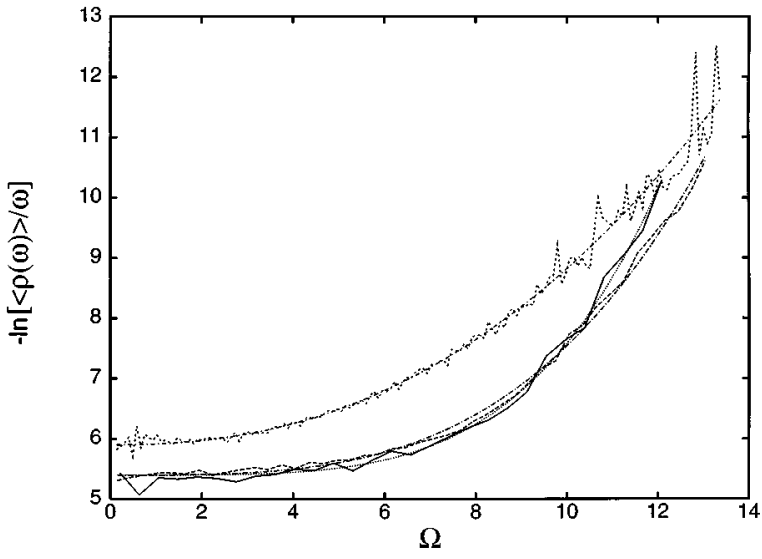

FIG. 2. Quantities in Fig. 1 as a function of scaled frequency $\Omega ; T=0.50$ (solid/dotted line), $T=1.0, T=5.0$ (uppermost curves).

regard [e.g., $\left.\omega^{4} /\left(1+\omega^{2}\right)\right]$ still gave poor fits. A very good and physically illuminating scheme has finally emerged. The starting point is

$$
\left.-\ln \left[\left\langle\rho_{u}(\omega ; T)\right\rangle / \omega\right]=a_{1}(T)+\left[a_{2}(T) \Omega\right]^{a 3(T)},\right\urcorner
$$

where we introduce the scaled frequency, $\Omega=\omega / \sqrt{ } T$, suggested by the exponents in Eqs. (2.5) and (2.9). The density of states is not a function of $\Omega$ only, and we cannot collapse the data from different $T$ onto a master plot with introduction of $\Omega$. Nevertheless the curves at different $T$ are much more similar viewed as functions of $\Omega$; this is demonstrated in Figs. 1 and 2, which also include the fits in their final form.

In the following $a_{1}(T)$, which determines a multiplicative constant in $\left\langle\rho_{u}(\omega ; T)\right\rangle$, is not so interesting and we focus on the second and third parameters. Fitting our simulation data with Eq. (3.1) leads to results for $a_{2}(T)$ shown in Fig. 3. The weak variation of $a_{2}(T)$, which increases by a factor of $\sim 1.6$ while $T$ increases by $\sim 24$, is clearly a consequence of the use of the scaled frequency. Since we want to minimize the number of parameters, it would be desirable to be able to treat $a_{2}(T)$ as a temperature independent constant, $a_{2}$. More

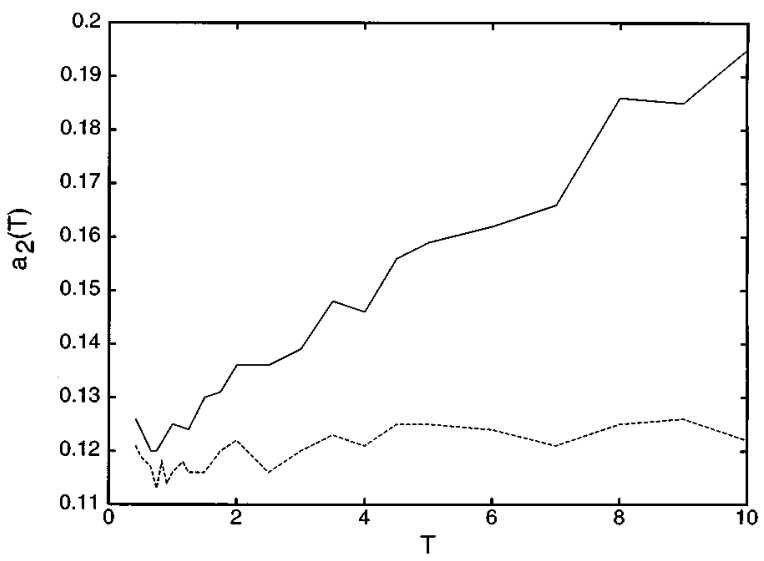

FIG. 3. Parameter $a_{2}(T)$ for fit to raw data(solid line) and for converged fit to Boltzmann factor only. 


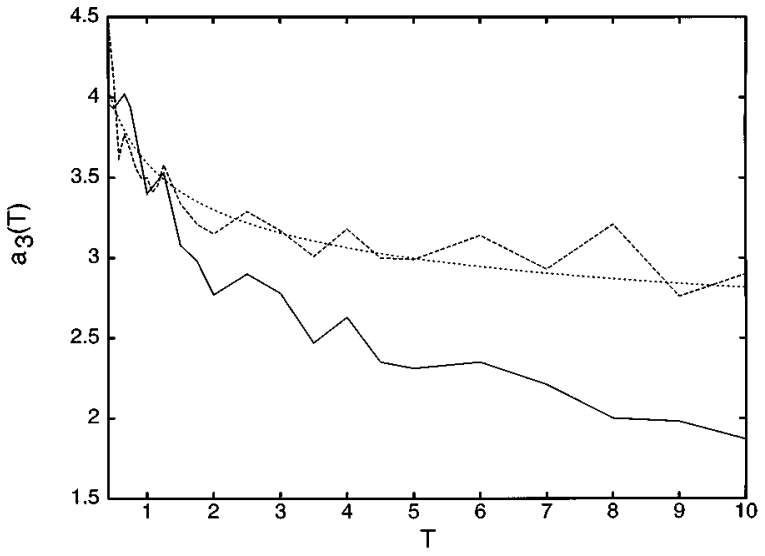

FIG. 4. Parameter $a_{3}(T)$ for fit to raw data (solid line), for converged fit to Boltzmann factor only (large dashes), and Eq. (3.5b).

importantly, a constant $a_{2}$ has physical implications, as we discuss later. Further refinement of the fit procedure makes this possible.

The values of $a_{3}(T)$ are shown in Fig. 4. The exponent is $\sim 4$ at the lowest supercooled temperatures and falls with increasing $T$. We expect that the high- $T$ value of $a_{3}(T)$ is 2 , corresponding to Arrhenius behavior. Before $T=10$ is reached, however, $a_{3}(T)$ drops below 2 , reaching $a_{3}=1.87$ at $T=10$. This behavior, while initially troubling, is in fact the key to understanding the $\omega, T$ dependence of the density of unstable modes.

According to Eq. (2.3), $\left\langle\rho_{u}(\omega)\right\rangle$ reaches a well defined high- $T$ limit. In this limit the Boltzmann factor is irrelevant and we are seeing properties of the potential surface only. There will be a corresponding contribution to the RHS of Eq. (3.1), which must be removed to obtain the Boltzmann factor; interpreting the entire exponent as if it were a Boltzmann exponent will lead to confusion. Defining the RHS as $a_{1}(T)+x p(\omega, T)$ we therefore write

$$
x p(\omega, T)=x G(\omega, T)+x_{\infty}(\omega),
$$

and the Boltzmann factor exponent $x G(\omega, T)$ defined by

$$
G(\omega, T)=\exp [-x G(\omega, T)]
$$

governs relaxation in supercooled liquids.

Both $x G(\omega, T)$ and $x_{\infty}(\omega)$ are found with an iterative application of Eq. (3.1). In a first approximation, it is assumed that $x_{\infty}(\omega)=x p(\omega, T=20)$; we thus repeat our initial fits, only $x p(\omega, T=20)$ is subtracted from the raw data for $-\ln \left[\left\langle\rho_{u}(\omega, T)\right\rangle / \omega\right]$. The first benefit of the method appears in a nearly constant $a_{2}(T)$ for $5>T>0.42 ; a_{2}(T)$ begins to vary at higher $T$ but that is a consequence of being at an early step (final version shown in Fig. 3) in the iteration with an unfinished estimate of $x_{\infty}(\omega)$. It is indeed possible to construct fits, with minimal loss of quality, using a $T$-independent $a_{2}$, which we choose as the median of $a_{2}(T)$ for $10>T$ $>0.42$. Using that value $\left(a_{2}=0.117\right.$ at this point $) a_{3}(T)$ is then redetermined, and the new $a_{3}(T)$ show a more systematic, $\neg$ less $\neg$ noisy $\neg$ variation. $\neg \mathrm{We} \neg$ now $\neg$ assume $\neg$ that

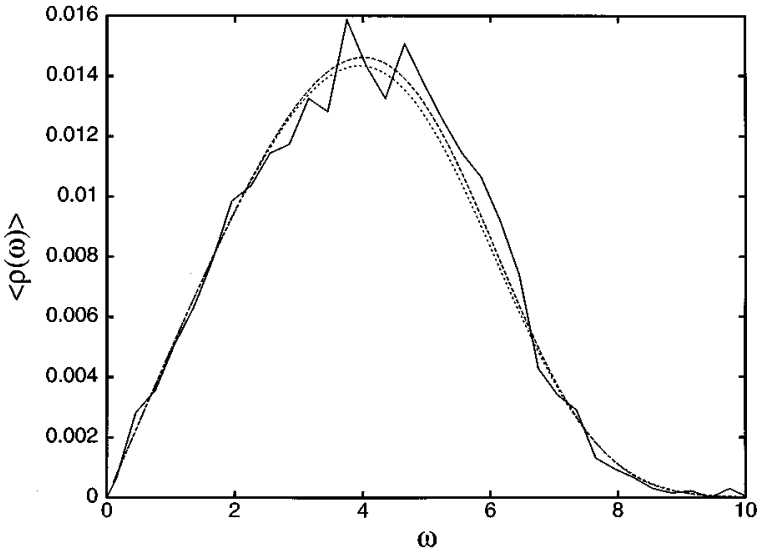

FIG. 5. Simulated unstable density of states at $T=0.50$ (solid line), converged fit using Eq. (3.5b) with numerically determined parameter $c_{3}$ (large dashes), two parameter fit using calculated $c_{3}=\ln 2 / \ln T_{\mathrm{FI}}$.

$a_{3}(T)$ - describing the Boltzmann factor only-should reach an asymptotic value of 2 and fit it, for $10>T>0.42$, to the function,

$$
\left.a_{3}(T)=2+\left(T_{\mathrm{Fl}} / T\right)^{c 3},\right\urcorner
$$

which again was found after many other tries. Here we introduce $T_{\mathrm{Fl}}$, one of the three physically significant temperatures in our formulation of supercooled dynamics; it is the crossover temperature below which stronger than Arrhenius behavior sets in due to the dominant role of barrier height fluctuations (Fl), or "nonuniform roughness", 11 of the potential energy surface, hereafter referred to as the "landscape.'

From Eq. (3.4) we calculate $a_{3}(20)$ and thus, with the median $a_{2}, x G(\omega, 20)$. Subtracting this from $x p(\omega, T=20)$ leads to a second approximation to $x_{\infty}(\omega)$, and now the iterative procedure is apparent. The third approximation has converged to within the noise, leading to our final results,

$$
\begin{aligned}
& -\ln \left[\left\langle\rho_{u}(\omega ; T)\right\rangle / \omega\right]=a_{1}(T)+x_{\infty}(\omega)+(0.120 \Omega)^{a 3(T)}, \\
& a_{3}(T)=2+(4.95 / T)^{0.29}, \neg \\
& x_{\infty}(\omega)=0.0114 \omega^{1.55}-0.000064 \omega^{2.67}, \neg
\end{aligned}
$$

thus $T_{\mathrm{Fl}}=4.95$. The final $a_{2}(T)$, and $a_{3}(T)$ along with Eq. (3.5b), are in Figs. 3 and 4. The constancy of $a_{2}(T)$ generated by the iterative fit is striking, and $a_{3}(T)$ is now quite plausibly equal to two in the high- $T$ limit. Note that, although we fit $a_{3}(T)$ with Eq. (3.4b) to estimate the $T=20$ Boltzmann factor, the values of $a_{3}(T)$ were unconstrained in the subsequent fits. Some representative $\left\langle\rho_{u}(\omega, T)\right\rangle$ and their fits are shown in Figs. 5-7; $a_{3}(T)$ is taken from Eq. (3.5b), it is not the optimum $a_{3}$, but the fits are good. Figure 8 displays $x_{\infty}(\omega)$; for $40>\omega>5$ it is quite linear. Of course it eventually becomes unphysically negative, for $\omega>100$, but this is an artifact. The numerical value of $x p(\omega, 20) \sim \omega^{1.55}$ arises from the sum of a nearly linear $x_{\infty}(\omega)$ with a slightly stronger than quadratic Boltzmann exponent. Exponential decay of $\left\langle\rho_{u}(\omega, T)\right\rangle$ in the high- $T$ limit has intriguing ${ }^{11}$ physical implications. 


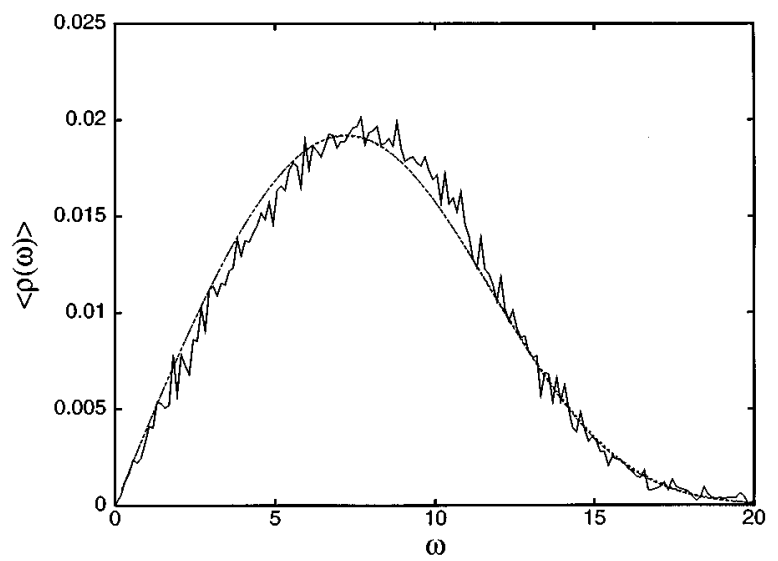

FIG. 6. As Fig. 5 for $T=2.00$.

\section{PHYSICAL INTERPRETATION OF THE FIT; DIFFUSION}

In the high- $T$ limit the cummulant expansion of $\langle\exp (-\beta E)\rangle_{\omega}$ must be rapidly convergent and we have

$$
G(\omega, T)=\exp (-\beta\langle E(\omega, T=\infty)\rangle) ;
$$

comparison with the high- $T$ limit of the fit yields

$$
\langle E(\omega, T=\infty)\rangle=a_{2}^{2} \omega^{2} . \neg
$$

In the original form of the fit, with $a_{2}(T)$, Eq. (4.2) would contain $a_{2}(T=\infty)$, and the quantity, $a_{2}(T)^{2} \omega^{2}$, would have no particular significance. The remarkable constancy of $a_{2}$ which we found, and the resulting fit with constant $a_{2}$, thus has two important physical implications. First, it suggests that the averaged barrier height for a given $\omega$ is $T$-independent; changes in the landscape as $T$ decreases increase the fluctuations leaving the mean unchanged. Second, the mean appears prominently in $G(\omega, T)$ even at $T$ where the fluctuations are dominant; the mean plays an important role in expressing the fluctuations.

Terms in the cummulant expansion after the first contain the fluctuations, so the only way $G(\omega, T)$ can attain a nonArrhenius fractional form is by fluctuation domination requiring a resummation of the cummulant series. Since $T<T_{\mathrm{Fl}}$ is the condition for non-Arrhenius behavior, we conclude that, for $T<T_{\mathrm{Fl}}$ the landscape is dominated by fluctuations or is "nonuniformly rough," while $T>T_{\mathrm{Fl}}$ is the regime of uniform roughness. This is in accord with the views ${ }^{11}$ of Stillinger. In sum, $a_{2}$ determines the mean energy, and $T_{\mathrm{Fl}}$ is the crossover temperature, or energy, for uniform to nonuniform roughness.

From Eq. (2.8), the exponential $T$-dependence (not power law contributions) of $D(T)$ is predicted to be $G\left(\omega_{c}, T\right)$. In the absence of fluctuations, Eq. (4.2) would yield the minimum barrier, $E_{\min }=a_{2}^{2} \omega_{c}^{2}$. Because of fluctuations, there will exist barriers with $E<E_{\text {min }}$, but we expect that the distribution of barriers will fall off very sharply for $E<E_{\min }$, and that $E_{\min }$ is an estimate of the smallest usable barrier. The exponential $T$-dependence of $D(T)$ is then [Eq. (3.5)].

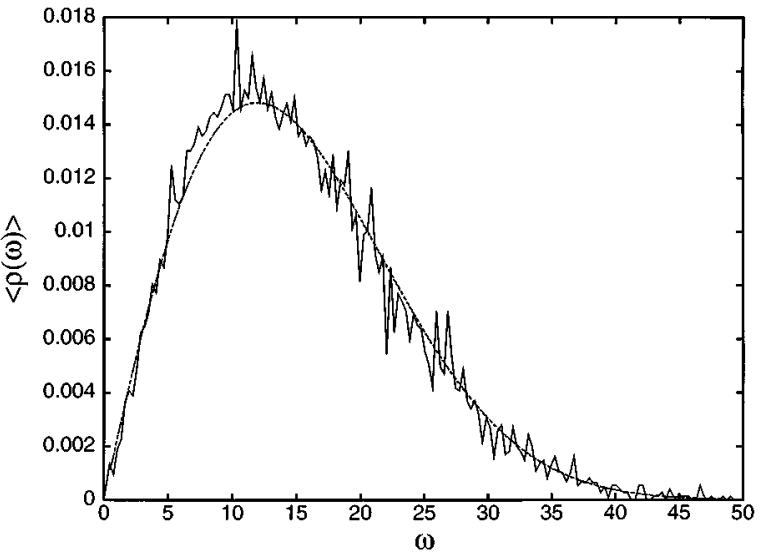

FIG. 7. As Fig. 5 for $T=10.00$.

$$
D(T) \sim \exp \left[-\left(T_{\min } / T\right)^{a 3(T) / 2}\right]
$$

(exponential behavior only)

and the significance of $T_{\min }$ for diffusion is clear. For $T>T_{\min }$, the exponent is small, strong $T$-dependence cannot occur, and $D(T)$ will be power law. For $T<T_{\min }$ exponential $T$-dependence sets in, and $T_{\min }$ is the crossover $T$ for power law to exponential $T$ dependence. Physically, for $T>T_{\min }$ the system can always find a barrier with $E<T$, so activated barrier crossing is unnecessary, and conversely. Mode coupling theory ${ }^{12}$ in its original form predicts that $D$ should vanish at a critical temperature $T_{x}$ as a power law $\left(T-T_{x}\right)^{\alpha}$, and later versions identify $T_{x}$ as a temperature for crossover to activated behavior. Thus $T_{x}$ and $T_{\min }$ are related empirically, but the physical pictures seem different.

The importance of the minimum barrier energy illustrates the care needed in interpreting the $T$-dependence of diffusion, even given the idea that barrier crossing is the governing process. A plausible intuitive guess for $D(T)$ would be $\exp (-\langle E\rangle / T)$, where $\langle E\rangle$ is the mean barrier en-

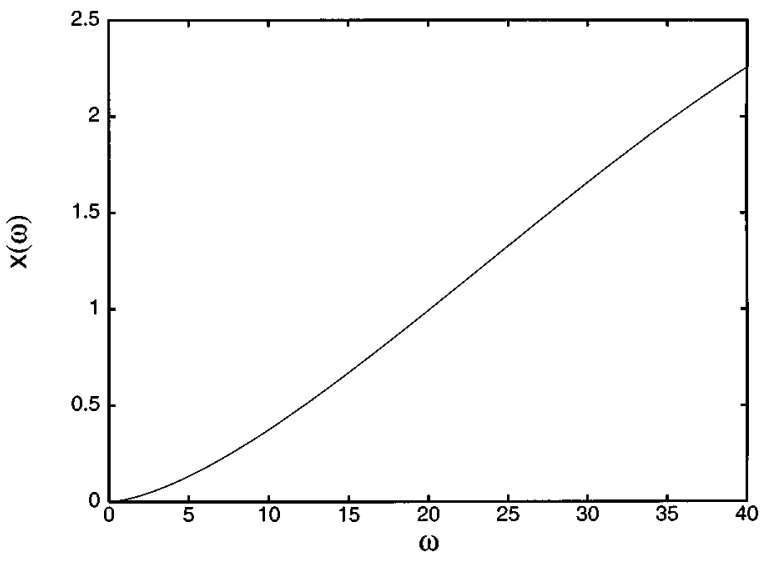

FIG. 8. Frequency dependence of the negative of the numerically determined exponent for the unstable density of states in the infinite temperature limit. 
ergy. The high $T$ limit of Eq. (4.3) is $\exp \left(-T_{\min } / T\right)$, however, indicating that the minimum energy, not the mean, is the activation energy.

In studies of supercooled liquids it is often useful to refer temperature to the glass transition temperature $T_{G}$, $T \rightarrow T / T_{G}$. Eqs. (3.5) and (4.3) still hold with all the $T$ referred to $T_{G}$. With the new convention and the estimates from Ref. 4, $T_{G} \sim 0.33$, and $\omega_{c} \sim 5$, the parameters for unit density $\mathrm{LJ}$ are $T_{\min }=1.08, T_{\mathrm{Fl}}=14.85$. The unusual behavior of this system is succinctly summarized by these numbers; $\mathrm{LJ}$ is in the fluctuation-dominated, non uniformly rough region of the potential surface for a very broad $T$-range ( $T$ $<14.85$ ) but for the most part this is irrelevant to diffusion; $T_{\min }$ is so close to unity that only just above the glass transition does activated barrier crossing become important. The presence of a nonuniformly rough landscape will not lead to stronger than Arrhenius behavior, or for that matter any exponential behavior, if barriers with $E<T$ are readily available.

The least obvious parameter to interpret is $c_{3}$, which governs the abruptness of the increase of $a_{3}(T)$ from its limiting high- $T$ value of two as $T$ falls below $T_{\mathrm{Fl}}$. Now, we do not wish to focus on LJ; it is our hope that the ideas developed here apply to supercooled liquids in general, with different substances characterized by different values of the parameters. For a full understanding of the parameters, it is necessary to see how they vary from substance to substance. Thus, we have used Eq. (4.3) to fit $\eta^{-1}$, the inverse of the shear viscosity (data taken from Ref. 6), for the prototypical "fragile" liquid, ortho terphenyl. Recent work ${ }^{14}$ has shown that the Stokes-Einstein law, $D \eta \sim T$, breaks down close to the glass transition, but it will serve as a first approximation to the exponential $T$-dependence. A good fit results, with $T_{\min }=5.29, T_{\mathrm{Fl}}=1.51$, and again the parameters nicely summarize the behavior of OTP. Exponential behavior is visible for $T<5.29$, with a higher minimum barrier (relative to $T_{G}$ ) than LJ, and the Arrhenius-non-Arrhenius crossover at $T=1.51$ is such that both types of $T$-dependence are easily observed before the glass transition is reached.

In $\mathrm{LJ}, D(T)$ is very close to a $\mathrm{ZB}$ law just above the glass transition; $a_{3}\left(T_{G}\right) / 2=2.09$. It is suggestive that the non-Arrhenius behavior in the fluctuation dominated region follows a standard model, but this is by no means built into the fitting function; there are no constraints on $a_{3}\left(T_{G}\right)$. Thus it is extremely gratifying to note that, for OTP, $a_{3}\left(T_{G}\right) / 2=2.21$, basically ZB again for the deeply supercooled liquid. One cannot help speculate that, in general, $a_{3}\left(T_{G}\right) / 2 \sim 2$. The evidence is for a value slightly greater than two, but we suggest that a physically appealing, numerically accurate fit may be had with $a_{3}\left(T_{G}\right) / 2 \equiv 2$, in which case $c_{3}$ is determined, $c_{3}=\ln 2 / \ln T_{\mathrm{F} 1}$. With this relation the fit contains two parameters only, assuming that $T_{G}$ is available separately. Fits using the calculated $c_{3}$ are shown in Figs. 5-7, and quality is comparable to that obtained with the "true" $c_{3}$. In the current viewpoint, ZB behavior is the consequence of fully developed barrier height fluctuations, and $c_{3}$ simply adjusts itself so that, irrespective of $T_{\mathrm{Fl}}, \mathrm{ZB}$ is attained at the glass transition.

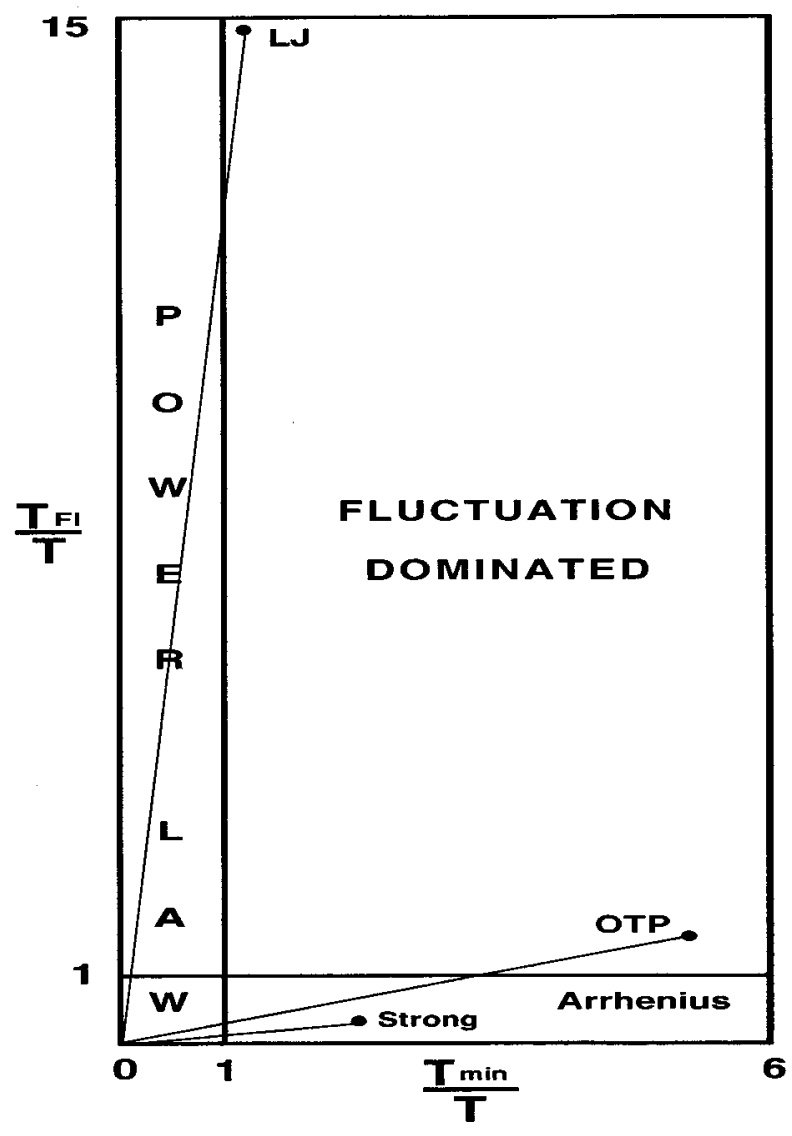

FIG. 9. Two parameter classification of liquids. $T$-dependence of relaxation is represented by a straight line passing through the four possible physically distinct regions in $\left[\left(T_{\min } / T\right),\left(T_{\mathrm{FI}} / T\right)\right]$ plane. LJ, OTP, and a generic strong liquid are shown.

Fragile liquid behavior is believed ${ }^{6}$ to be a consequence of nondirectional, van der Waals intermolecular interactions. Thus, rare gas liquids, not OTP, should be the ultimate fragile liquids. Of course the supercooled states of such simple liquids cannot be studied in the laboratory, but the logic applies to our simulation of LJ. At first, it would not appear possible that a liquid exhibiting power-law $D(T)$ down to $T / T_{G}=1.08$ could be an exemplar of "fragility." However, with the expanded viewpoint available through $\left\langle\rho_{u}(\omega, T)\right\rangle$ $\mathrm{LJ}$ is clearly seen to be an exceptionally fragile liquid, with nonuniformly roughness over the enormous range 14.85 $>T / T_{G}>1$. This characteristic "fragile" potential surface topology does not manifest itself in a stronger than Arrhenius $D(T)$ until $T<T_{\min }$, but the correlation between the nature of the intermolecular interactions and the topology still holds. Apparently the most meaningful correlation is between the interactions and $T_{\mathrm{Fl}}$, the extent of the nonuniformly rough region of the landscape, which may or may not manifest itself in $D(T)$ depending on the value of $T_{\min }$. The density of states is a more sensitive indicator of fragility than is $D(T)$, conveying useful information about the landscape at temperatures where $D(T)$ is an uninformative power law.

A schematic classification of strong and fragile liquids is possible based upon the two temperatures (relative to $T_{G}$ ), 
$T_{\mathrm{Fl}}$, and $T_{\min }$. Consider a two-dimensional Cartesian coordinate frame where the $x$ axis is $T_{\min } / T$ and the $y$ axis $T_{\mathrm{Fl}} / T$. The state of a liquid vs $T$ is represented as a straight line in the plane, starting at zero, with slope $=T_{\mathrm{Fl}} / T_{\min }$ and terminating at a point determined by $T_{G}$. Figure 9 represents unit density LJ and OTP in this scheme, along with a hypothetical strong liquid. Sokolov ${ }^{13}$ has observed that the ratio, $T_{x} / T_{G}$, is closer to unity in fragile liquids than in strong. Identifying $T_{\min }$ with the mode coupling $T_{x}$, this makes obvious sense in terms of Fig. 9.

The LJ simulations are at constant density, while experiments on OTP are at constant pressure. Our hope is that constant $T$ or constant $P$ conditions manifest themselves in different values for the parameters, but the basic ideas presented here hold for both cases; verifying this is a current research project. Fig. 9 describes " $P=1$ atm" OTP and unit density LJ, not these substances in general. Further research on the pressure and density dependence of $\left\langle\rho_{u}(\omega, T)\right\rangle$ should allow an INM treatment of the $P$ dependence of fragility, another topic ${ }^{6}$ of contemporary interest.

\section{SUMMARY}

The $T$-dependence of relaxation times in liquids has been ${ }^{6}$ exhaustively studied, and is currently ${ }^{13,14}$ a field of active investigation. Crossover, from power law to Arrhenius and from Arrhenius to stronger exponential $T$-dependence occupies a central role in these studies. On the other hand, INM theory is quite new, and we have only here, for the first time, analyzed the $T$-dependence of $\left\langle\rho_{u}(\omega, T)\right\rangle$ over a range broad enough to observe crossover. We find that the $T$-dependence of $\left\langle\rho_{u}(\omega, T)\right\rangle$ mirrors that of $D(T)$.

The behavior of $\left\langle\rho_{u}(\omega, T)\right\rangle$ is readily interpreted in terms of the potential energy landscape, and thus an INM theory of $D(T)$ provides an excellent link between the landscape and $D(T)$; we believe that INM is uniquely suited to provide such a connection. Much will be learned by simply repeating this work for different densities and chemical substances, and by analyzing constant pressure quenches. INM also connects very well with the views outlined by Sokolov, ${ }^{13}$ who divides 'mesoscopic' dynamics into 'relaxationlike' contributions from anharmonic double well potentials and 'quasi-local' harmonic modes, and notes that that the mesoscopic dynamics, remarkably, correlate with structural relaxation which can be 10 orders of magnitude slower. This picture corresponds explicitly to double well $\mathrm{Im}-\omega$ INM, harmonic $\operatorname{Re}-\omega$ INM, and the calculation of $D(T)$ (structural relaxation) from the INM. Similarly, INM theories can provide ${ }^{15}$ new insights into other aspects of supercooled liquids, such as ${ }^{14}$ the role of spatial heterogeneity.

\section{ACKNOWLEDGMENTS}

This research was supported by NSF Grant No. CHE9415216. We thank Dr. Susan Keyes for assistance with the figures.

${ }^{1}$ G. Seeley and T. Keyes, J. Phys. Chem. 91, 5581 (1989); R. M. Stratt, Acc. Chem. Res. 28, 201 (1995).

${ }^{2}$ M. Goldstein, J. Chem. Phys. 51, 3728 (1969).

${ }^{3}$ R. Zwanzig, J. Chem. Phys. 79, 4507 (1983).

${ }^{4}$ T. Keyes, J. Chem. Phys. 101, 5081 (1994).

${ }^{5}$ S. Bembenek and B. B. Laird, Phys. Rev. Lett. 74, 936 (1995); J. Chem. Phys. 104, 5199 (1996).

${ }^{6}$ C. A. Angell, Science 267, 1924 (1995).

${ }^{7}$ R. Zwanzig, Proc. Natl. Acad. Sci. (USA) 85, 2029 (1988); H. Bassler, Phys. Rev. Lett. 58, 767 (1987); an earlier derivation is given by P. G. De Gennes, J. Stat. Phys. 12, 463 (1975).

${ }^{8}$ B. Madan, G. Seeley, and T. Keyes, J. Chem. Phys. 94, 6762 (1991).

${ }^{9}$ U. Zurcher and T. Keyes, Phys. Rev. E (in press).

${ }^{10}$ G. V. Vijayadamodar and A. Nitzan, J. Chem. Phys. 103, 2169 (1995).

${ }^{11}$ F. H. Stillinger, Science 267, 1935 (1995), and references therein.

${ }^{12}$ W. Goetze, in Liquids, Freezing, and the Glass Transition, edited by J. P. Hansen, D. Levesque, and J. Zinn-Justin (Elsevier, NY, 1991).

${ }^{13}$ A. Sokolov, Science 273, 1675 (1996).

${ }^{14}$ M. Cicerone and M. Ediger, J. Chem. Phys. 103, 5684 (1995).

${ }^{15}$ U. Zurcher and T. Keyes (unpublished). 
Journal of Chemical Physics is copyrighted by AIP Publishing LLC (AIP). Reuse of AIP content is subject to the terms at: http://scitation.aip.org/termsconditions. For more information, see http://publishing.aip.org/authors/rights-and-permissions. 